\title{
Biographie des auteurs
}

Abdérafi Charki est enseignant-chercheur à l'Institut des Sciences et Techniques de l'Ingénieur d'Angers (ISTIA) (école d'ingénieurs de l'Université d'Angers). Il y enseigne la métrologie et la qualité. Sa recherche au sein du LASQUO (LAboratoire de Sûreté de fonctionnement, Qualité et Organisation) est axée sur la fiabilité de systèmes complexes. Il intervient également en tant qu'expert et évaluateur dans les laboratoires d'essais, d'analyses et d'étalonnages. Il est président du CAFMET (Comité Africain de Métrologie, http://www.ac-metrology.com). Il est par ailleurs éditeur en chef du journal «International Journal of Metrology and Quality Engineering " (http://www.metrology-journal.org).

Patrick Gérasimo est directeur médical du Laboratoire d'Analyse Médicale d'Électricité de France. Il est évaluateur technique au Comité Français d'Accréditation (COFRAC) et membre de la Commission Technique d'Accréditation du COFRAC Electricité Rayonnement. Auteur de 53 publications et éditions scientifiques, il a participé à la mise en place des systèmes de management de la qualité et préparé à l'accréditation les différents laboratoires qu'il a dirigés comme le Laboratoire de Contrôle Radiotoxicologique du Service de Protection Radiologique des Armées. En juillet 2000, dans le cadre du Service de Santé des Armées, il a élaboré le Dossier Technique sur l'Uranium Appauvri remis au ministre de la Défense Alain Richard. 
Mohamed El Mouftari est ingénieur radiochimiste au Laboratoire d'Analyses Médicales d'EDF.

Yvon Mori a été responsable d'un laboratoire d'essais et d'évaluation à Thales Underwater Systems à Sophia Antipolis. Il a enseigné au CNAM de Nice en électronique B3 pendant quinze ans et à l'IUT Génie Télécoms Réseaux de Nice Sophia Antipolis pendant huit ans. Il forme aujourd'hui les ingénieurs des grandes sociétés en mécanique des vibrations et des chocs, ainsi qu'en compatibilité électromagnétique. Il est auditeur expert auprès du Cofrac pour le programme 38 et président du Groupe Régional de la Cote d'Azur de la SEE.

Christian Sauvageot est responsable technique de l'unité Microbiologie du Laboratoire du Commissariat des Armées. Il a réalisé deux rapports remis au Ministère de la Défense concernant la métrologie des balances et la fertilité d'un milieu microbiologique. 